Proceedings

\title{
Nanotechnological Strategies for Administration of Poorly Soluble Neuroactive Drugs ${ }^{\dagger}$
}

\author{
Elisabetta Esposito ${ }^{1, *}$, Maddalena Sguizzato ${ }^{1}$, Markus Drechsler ${ }^{2}$, Paolo Mariani ${ }^{3}$, Viviana Trezza ${ }^{4}$, Rita Cortesi ${ }^{1}$ \\ and Claudio Nastruzzi ${ }^{1}$
}

Citation: Esposito, E.; Sguizzato, M.; Drechsler, M.; Mariani, P.; Trezza,

V.; Cortesi, R.; Nastruzzi, C.

Nanotechnological Strategies for

Administration of Poorly Soluble Neuroactive Drugs. 2021, 78, 21. https://doi.org/10.3390/IECP202008678

Published: 1 December 2020

Publisher's Note: MDPI stays neutral with regard to jurisdictional claims in published maps and institutional affiliations.

Copyright: () 2020 by the authors. Licensee MDPI, Basel, Switzerland. This article is an open access article distributed under the terms and conditions of the Creative Commons Attribution (CC BY) license (http://creativecommons.org/licenses/by/4.0/).
1 Department of Chemical, Pharmaceutical and Agricultural Sciences, University of Ferrara, 44121 Ferrara, Italy; sgzmdl@unife.it (M.S.); crt@unife.it (R.C.); nas@unife.it (C.N.)

2 Bavarian Polymer Institute (BPI) Keylab "Electron and Optical Microscopy", University of Bayreuth, 95440 Bayreuth, Germany; markus.drechsler@uni-bayreuth.de

3 Department of Life and Environmental Sciences, Polytechnic University of Marche, 60131 Ancona, Italy; p.mariani@staff.univpm.it

4 Department of Science, Roma Tre University, 00146 Rome, Italy; viviana.trezza@uniroma3.it

* Correspondence: ese@unife.it; Tel.: +39-0532-455230

+ Presented at the 1st International Electronic Conference on Pharmaceutics, 1-15 December 2020; Available online: https://iecp2020.sciforum.net/.

\begin{abstract}
Many neuroactive drugs are characterized by poor solubility, hampering their therapeutic potential and clinical research studies. For instance, the lipophilic molecules dimethylfumarate, retinyl palmitate, progesterone, and URB597 can be employed in the treatment of relapsing remitting multiple, early brain injury, learning deficits, and/or traumatic brain injuries. In this study, the possibility to encapsulate these drugs in lipid nanoparticles is investigated. Solid lipid nanoparticles and nanostructured lipid carriers have been produced by melt and ultrasonication of stearic triglyceride or a mixture of stearic triglyceride and caprylic/capric triglycerides. Mean diameters and morphology of lipid particles were studied by photon correlation spectroscopy, cryo-transmission electron microscopy, and x-ray diffraction, while encapsulation efficiency and in vitro drug release have been determined by HPLC. A behavioral study was conducted in rats to study the capability of lipid nanoparticles containing URB597 to alter behaviors relevant to psychiatric disorders after intranasal administration. In this regard, the nanoparticle surface has been modified by polysorbate 80 in order to obtain "stealth" nanoparticles. The nanoencapsulation strategy allowed increased drug solubility with respect to unphysiological solvent or solvent mixtures usually employed for animal and clinical studies. In particular, retinyl palmitate solubility in nanostructured lipid carriers has been increased up to eight-fold. Moreover, rat behavioral effects observed by nanoencapsulated URB597 administered intranasally suggest the therapeutic potential of this non-invasive route to treat social dysfunctions, such as autism.
\end{abstract}

Keywords: solid lipid nanoparticles; nanostructured lipid carriers; URB597; dimethyl fumarate; retynil palmitate; progesterone

\section{Introduction}

The administration of potentially effective therapeutics can be critical in the case of poorly soluble drugs. Indeed, the low bioavailability of unsoluble drugs hampers their use in clinical research [1]. Particularly, since many neuroactive drugs are lipid-soluble, research efforts are devoted to the development of specialized delivery systems suitable for their solubilization and administration to the central nervous system [2]. The nanoencapsulation strategy enables to (a) solubilize drugs with different physico-chemical properties, (b) control their release, (c) reduce dosage and number of drug administrations, 
and (d) improve drug targeting/biodistribution [3,4]. Particularly lipid-based nanoparticles can be regarded as biocompatible and biodegradable nanocarriers suitable for solubilization and delivery of poorly soluble drugs. Research efforts have led to the development of two main types of lipid nanoparticles characterized by different lipid matrixes. Namely, solid lipid nanoparticles (SLN) have an ordered matrix constituted of lipids in solid state at room temperature, while nanostructured lipid carriers (NLC) are characterized by a disordered matrix composed of solid and liquid lipid mixtures $[5,6]$. This study focuses on the production and characterization of SLN and NLC as carriers for poorly soluble drugs with potential in the treatment of neurological disorders. Particularly, dimethylfumarate, retinyl palmitate, progesterone, and URB597, have been studied, being effective in the treatment of relapsing remitting multiple, early brain injury, learning deficits and/or traumatic brain injuries [1]. A behavioral study was conducted in rats to study the capability of SLN containing URB597 to alter behaviors relevant to psychiatric disorders after intranasal administration. In this regard, the nanoparticle surface has been modified by polysorbate 80 , in order to obtain pegylated "stealth" nanoparticles suitable to escape the opsonization phenomenon.

\section{Experiments}

\subsection{Materials}

Tristearin, stearic triglyceride (tristearin), polyoxyethylenesorbitan monooleate, polysorbate 80 (P80), polyethylene glycol 400 (PEG400), dimethyl fumarate (DMF), retinyl palmitate (RP), progesterone (PRG) and [3-(3-carbamoylphenyl)phenyl] N-cyclohexylcarbamate (URB597) were purchased from Sigma-Aldrich, Merck (Darmstadt, Germany). The copolymer poly (ethylene oxide) (a) -poly (propylene oxide) $(b)(a=80, b=27)$ (poloxamer 188) was a gift of BASF ChemTrade GmbH (Burgbernheim, Germany). Miglyol 812 N, caprylic/capric triglycerides (miglyol) was a gift of Cremer Oleo Division (Witten, Germany).

\subsection{Nanoparticle Preparation}

SLN and NLC have been produced by melt and ultrasonication of stearic triglyceride or a stearic triglyceride-caprylic/capric triglycerides mixture [7]. In the case of SLN, the lipid phase (5\% with respect to the whole weight of the dispersion) was constituted of pure tristearin, while in the case of NLC a mixture of tristearin and miglyol in a 2:1 w/w ratio was employed. An aqueous poloxamer 188 solution $(2.5 \% \mathrm{w} / \mathrm{w})$ heated at $80{ }^{\circ} \mathrm{C}$ was added to the molten lipid phase. Afterwards, the mixture was emulsified at 15,000 rpm, $80^{\circ} \mathrm{C}$ for $1 \mathrm{~min}$, subjected to ultrasonication at $6.75 \mathrm{kHz}$ for $15 \mathrm{~min}$ and then let cooling at $25{ }^{\circ} \mathrm{C}$. Lipid nanoparticle dispersions were stored at room temperature. In the case of drug-containing nanoparticles, the drug was added to the molten lipid phase before adding the aqueous poloxamer 188 solution. To prepare pegylated nanoparticles (SLN/P80 and SLN/P80-URB597), P80 (20\% w/w with respect to the lipid weight) was added to the SLN dispersion during the preparation, namely after the ultrasonication step, when the dispersion was at $40{ }^{\circ} \mathrm{C}$. The stirring at $250 \mathrm{rpm}$ was maintained until the preparation was cooled down at room temperature (usually in $30 \mathrm{~min}$ ).

\subsection{Nanoparticle Characterization}

Mean diameters were studied by photon correlation spectroscopy (PCS), using a Zetasizer 3000 PCS (Malvern Instr., Malvern, UK) equipped with a 5-mW helium neon laser with a wavelength output of $633 \mathrm{~nm}$. Glassware was cleaned of dust by washing with detergent and rinsing twice with water for injections. Measurements were made at $25^{\circ} \mathrm{C}$ at an angle of $90^{\circ}$. Data were interpreted using the CONTIN method [8].

The morphology of lipid particles has been studied by cryo-transmission electron microscopy (cryo-TEM) vitrifying samples as previously described [6]. The obtained specimen was put in a Zeiss EM922Omega transmission electron microscope for imaging using a cryoholder (CT3500, Gatan, Pleasanton, CA, USA), keeping the sample temperature 
below $-175^{\circ} \mathrm{C}$ throughout the examination. Images were recorded digitally by a CCD camera (Ultrascan 1000, Gatan) using an image processing system (GMS 1.9 software, Gatan).

X-ray diffraction experiments were performed by a $3.5 \mathrm{~kW}$ Philips PW 1830 X-ray generator (Amsterdam, The Netherlands) equipped with a Guinier-type focusing camera (homemade design and construction, Ancona, Italy) operating with a bent quartz crystal monochromator $(\lambda=1.54 \AA$ A $)$. Diffraction patterns were recorded on GNR Analytical Instruments Imaging Plate system (Novara, Italy). Samples were held in a tight vacuum cylindrical cell provided with thin mylar windows. Diffraction data were collected at $37^{\circ}$. In each experiment, Bragg peaks were detected [1]. The few peaks in the low-angle region were indexed considering the different symmetries commonly observed in lipidic phases and the unit cell dimension of the phase, calculated from the averaged spacing of the observed peaks.

The encapsulation efficiency (EE) of drugs in SLN and NLC was determined by ultracentrifugation and HPLC [5,7]. Briefly, 500 microliters aliquot of SLN or NLC were poured into a centrifugal filter (Microcon centrifugal filter unit YM-10 membrane, NMWCO $10 \mathrm{kDa}$, Sigma-Aldrich, St. Louis, MO, USA) and centrifuged (Spectrafuge ${ }^{\mathrm{TM}}$ 24D Digital Microcentrifuge, Woodbridge, NJ, USA) for $20 \mathrm{~min}$ at $8000 \mathrm{rpm}$. The lipid phase in the upper section of the filter was then diluted in a 1:10 v/v ratio with methanol. After sample filtration by nylon syringe filters $(0.22 \mu \mathrm{m}$ pores $)$, the amount of drug has been analyzed by high-performance liquid chromatography (HPLC), as below reported. The EE was determined as follows:

$$
\mathrm{EE}=\mathrm{D} / \mathrm{T}_{\mathrm{D}} \times 100
$$

where $\mathrm{D}$ is the amount of drug encapsulated in SLN or in NLC and $\mathrm{T}_{\mathrm{D}}$ is the total content of drug.

HPLC analysis was carried out by an Agilent Zorbax Eclipse XBD-C18 column (Agilent Technologies, Santa Clara, CA, USA) $(15 \mathrm{~cm} \times 0.46 \mathrm{~cm})$ stainless steel packed with 5 $\mu \mathrm{m}$ particles, eluted at room temperature with different mobile phases. Samples of $50 \mu \mathrm{L}$ were injected through the rheodyne injector system fitted with $50 \mu \mathrm{L}$ fixed loop and compared with standards of known concentration. The mobile phase flow rate was $1 \mathrm{~mL} / \mathrm{min}$ and the composition was: acetonitrile/water $(25: 75, v / v)$, methanol/water $(70: 30, v / v)$, methanol, and methanol/water (80:20, v/v), for DMF, RP, PRG and URB597 respectively. Chromatograms were obtained with a UV detector set at $220 \mathrm{~nm}$ for DMF, $350 \mathrm{~nm}$ for RP, $254 \mathrm{~nm}$ for PRG and $280 \mathrm{~nm}$ for URB597. Analyses were conducted in triplicate, mean, and standard deviation values were calculated.

\subsection{In Vitro URB597 Release Studies}

Release kinetics of URB597 alternatively solubilized in PEG 400/P80/saline 5:5:90 $(v / v / v)$, in SLN/P80, or suspended in poloxamer 188 solution $(2.5 \% w / w)$ have been investigated by in vitro dialysis release studies [1]. Namely, $2 \mathrm{~mL}$ of solution/suspension (URB597 $0.2 \mathrm{mg} / \mathrm{mL}$ ) were put into a dialysis tube $(6 \mathrm{~cm}$ ) (molecular weight cut off 10,00012,000; Medi Cell International, England), then placed into $30 \mathrm{~mL}$ of receiving phase constituted of phosphate buffer $(100 \mathrm{mM}, \mathrm{pH} 7.4)$ and ethanol $(70: 30, v / v)$ and shaken in a horizontal shaker (MS1, Minishaker, IKA) at $175 \mathrm{rpm}$ at $37^{\circ} \mathrm{C}$. Samples of receiving phase were withdrawn at regular time intervals, analyzed by HPLC method as above described and replaced by fresh receiving mixture to maintain constant volume. The URB597 concentrations were determined 6 times in independent experiments and the mean values \pm standard deviations were calculated.

\subsection{In Vivo Behavioral Study}

Male adolescent Wistar Han rats (28-35 g at the time of the experiments; Charles River Laboratories, Écully, France) were housed in groups of six in a temperature-controlled $\left(20 \pm 1^{\circ} \mathrm{C}\right)$ vivarium and maintained under a $12 \mathrm{~h}$ light/dark cycle (07:00 AM-07:00 
PM h lights on). Food and water were available ad libitum. The experiments were approved by the Italian Ministry of Health (Rome, Italy) and performed in agreement with the guidelines released by the Italian Ministry of Health (D.L. 26/14) and the European Community Directive 2010/63/EU. Social behavior was assessed as previously described $[1,9,10]$. Two hours before testing, pairs of animals were treated by intranasal route with either SLN/P80-URB597 or SLN/P80 at the dose of $0.1 \mathrm{mg} / \mathrm{Kg}$. Drug dose and pre-treatment interval were based on literature. In preclinical studies, URB597 is usually dissolved in a mixture of plain PEG400/P80/saline and, administered by intraperitoneal (i.p.) injection $2 \mathrm{~h}$ before testing at the dose of $0.1 \mathrm{mg} / \mathrm{kg}$, has prosocial effects in rats $[9,10]$. Therefore, as additional control, other pairs of rats were treated with URB597 $(0.1 \mathrm{mg} / \mathrm{kg}$, i.p.) dissolved in PEG400/P80/saline or plain PEG400/P80/saline. The social behavior test consisted of placing two similarly treated animals into the test cage for $15 \mathrm{~min}$. The behaviors of the animals were recorded using a camera with zoom lens, video tape recorder, and television monitor. Behavior was assessed per pair of animals and analyzed by a trained observer who was unaware of treatment condition using the Observer XT software (Noldus, Wageningen, The Netherlands).

\section{Results}

\subsection{Nanoparticle Preparation}

In this study, four neuroactive drugs have been considered, being effective in the treatment of different CNS disorders. Namely, DMF and PRG employed to treat early or traumatic brain injuries [11,12]; RP for multiple sclerosis [13] and URB597 for animal models of anxiety, depression, and autism spectrum disorders [14] were studied. All drugs are characterized by poor aqueous solubility and slight solubility in ethanol. Indeed, $\log p$ values are $0.72,11.62,3.87$, and 4.03 for DMF, RP, PRG, and URB597, respectively. Since the use of ethanol solutions can induce acute intravenous toxicity in animals and behavioral effects in humans by inhalation [1], lipid nanoparticles were designed as alternative strategies to administer the drugs. SLN and NLC were prepared by a method based on the emulsification of lipids in an aqueous poloxamer solution by hot homogenization and ultrasonication, as previously reported [5,7], using pure tristearin or a mixture of tristearin and miglyol for SLN and NLC respectively. The drugs were added to the molten lipids just before poloxamer solution addition. In the case of SLN produced in the absence and in the presence of URB597, polysorbate 80 has been added to the nanoparticles before cooling, in order to create "stealth" nanoparticle able to escape to opsonization process after in vivo administration. The PEG chains of the hydrophilic portion of P80 are thought to protrude into the aqueous phase out from SLN surface, protecting the SLN from the environment, while the lipophilic part of P80 behaves as a lipid anchor.

\subsection{Nanoparticle Characterization}

The effect of drug presence has been investigated on size distribution, morphology, and inner organization of SLN and NLC. Table 1 reports $Z$ average and Dispersity of SLN and NLC, as obtained by PCS. Mean diameters ranged between 130 and $262 \mathrm{~nm}$, dispersity was always below 0.3 , indicating homogeneous size distribution, both for SLN and NLC. The pegylation process induced a slight increase in SLN and SLN-URB597 mean diameters. The $\mathrm{X}$-ray scattering low-angle region analysis suggested the lamellar morphology of nanoparticles. Particularly, NLC displayed slightly larger interlamellar distances with respect to the corresponding SLN, as summarized in Table 1, suggesting an expanded packing of nanoparticle matrix occurring in the presence of miglyol, which is characterized by a liquid consistency. 
Table 1. Size parameters, structural organization, and encapsulation efficiency of nanoparticles.

\begin{tabular}{ccccc}
\hline Nanoparticle & Z Average $(\mathbf{n m})$ & Dispersity & X-ray Diffraction Interlamellar Distance (nm) & EE $^{\mathbf{1}} \mathbf{( \% )}$ \\
\hline SLN & $147 \pm 52$ & $0.26 \pm 0.01$ & 3.74 & - \\
SLN/P80 & $176 \pm 25$ & $0.25 \pm 0.08$ & 4.55 & - \\
NLC & $179 \pm 55$ & $0.27 \pm 0.01$ & 4.55 & - \\
SLN-DMF & $254 \pm 28$ & $0.25 \pm 0.01$ & 3.81 & $85.2 \pm 0.1$ \\
NLC-DMF & $195 \pm 20$ & $0.25 \pm 0.01$ & 4.49 & $71.2 \pm 0.3$ \\
SLN-RP & $192 \pm 46$ & $0.27 \pm 0.04$ & 4.60 & $45.1 \pm 0.1$ \\
NLC-RP & $129 \pm 22$ & $0.24 \pm 0.03$ & 4.68 & $48.3 \pm 0.2$ \\
SLN-PRG & $160 \pm 70$ & $0.30 \pm 0.02$ & 3.80 & $72.5 \pm 0.3$ \\
NLC-PRG & $187 \pm 64$ & $0.29 \pm 0.03$ & 4.52 & $73.8 \pm 0.1$ \\
SLN-URB597 & $246 \pm 33$ & $0.28 \pm 0.03$ & 4.50 & $93.0 \pm 0.2$ \\
SLN/P80-URB597 & $273 \pm 20$ & $0.30 \pm 0.03$ & 4.48 & $93.0 \pm 0.1$ \\
NLC--URB597 & $242 \pm 45$ & $0.29 \pm 0.01$ & 4.69 & $92.8 \pm 0.3$ \\
\hline
\end{tabular}

1: percentage $(w / w)$ of drug in the whole dispersion with respect to the total amount used for the preparation. Data are the means \pm SD of 6 independent batches. Adapted from [1].

The shape of nanoparticles was in general discoid both for SLN and NLC, they appear as low electron density ellipses when viewed from the top and electron-dense rods when edge-on viewed (Figure 1).
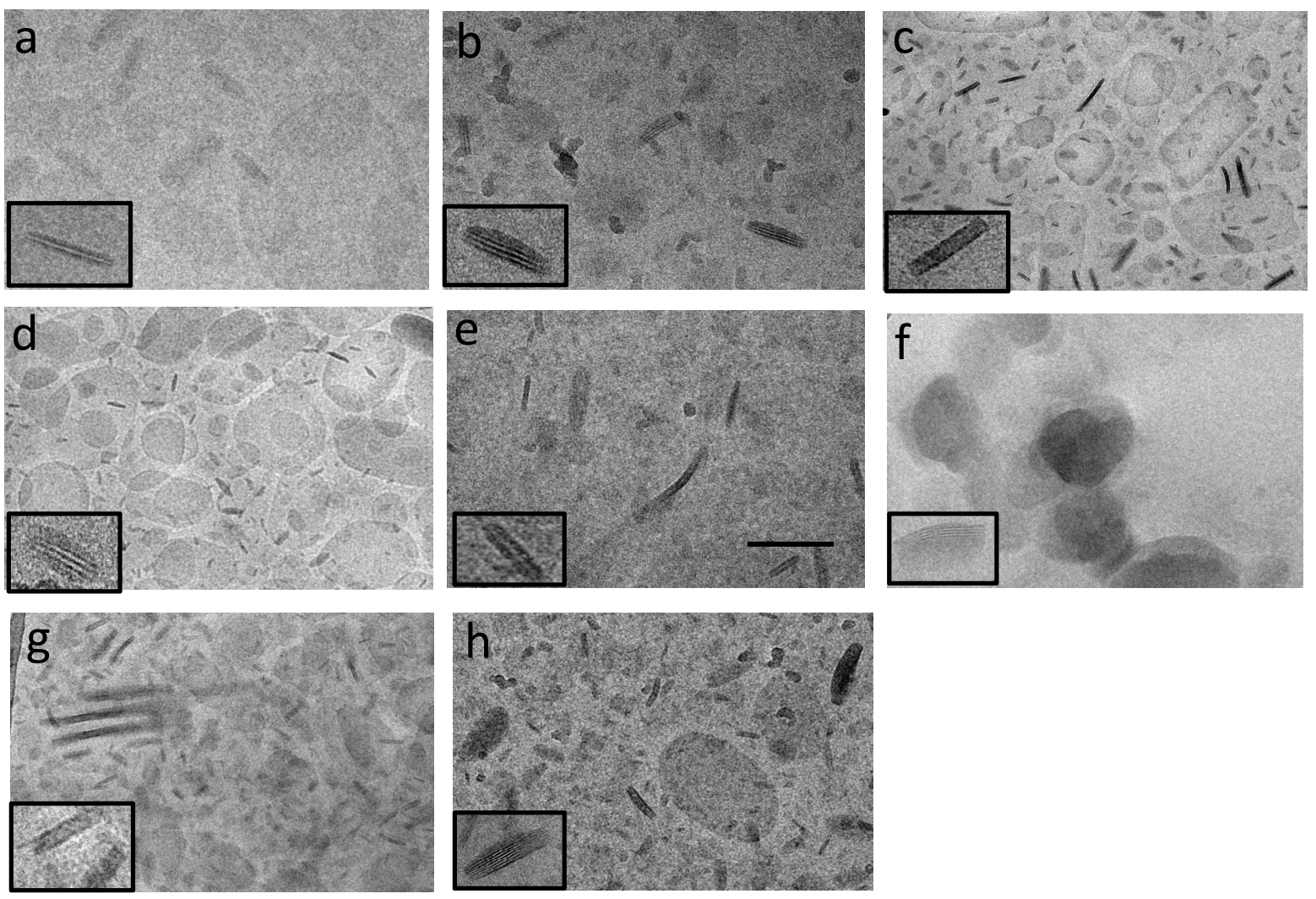

Figure 1. Cryo-TEM edge-on-viewed images of solid lipid nanoparticles (SLN) (a-d) and nanostructured lipid carriers $(\mathrm{NLC})(\mathbf{e}-\mathbf{h})$ containing different drugs, namely dimethyl fumarate (DMF) $(\mathbf{a}, \mathbf{e})$, retinyl palmitate $(\mathrm{RP})(\mathbf{b}, \mathbf{f})$, progesterone $(\mathrm{PRG})(\mathbf{c}, \mathbf{g})$, URB597 (d,h). Bar corresponds to $150 \mathrm{~nm}$. For nanoparticle acronym and composition, please refer to experimental section. 
The presence of the drug did not induce appreciable differences on the morphology of SLN and NLC, only in the case of NLC-RP the particles appear faint when imaged by cryo-TEM, possibly indicating a disorganization of nanoparticle internal structure due to the presence of RP.

The ability of SLN and NLC to encapsulate the drugs, expressed as EE value and reported in Table 1, follows the order URB $>$ DMF $>$ PRG $>$ RP. EE values in SLN and NLC were similar, except for DMF, displaying a higher EE value within SLN.

The solubility of drugs in SLN and NLC was compared to solubility in PEG 400/P80/saline, a standard vehicle often used in clinical and preclinical studies [1]. As summarized in Figure 2, in all cases, nanoparticles increased the solubility of drugs with respect to PEG400/P80/saline. The solubility improvement was particularly marked in the case of RP and PRG. Indeed, RP solubility in SLN and NLC increased 8/8.5-fold with respect to PEG400/P80/saline, while PRG solubility underwent a 4.3/4.3-fold increase in SLN and NLC, respectively. On the other hand, URB597 solubility was the lowest, passing from $0.12 \mathrm{mg} / \mathrm{mL}$ in PEG 400/P80/saline to $0.18 \mathrm{mg} / \mathrm{mL}$ upon nano-encapsulation (1.5-fold increase). Nonetheless, due to the URB597 interesting pharmacological profile (i.e., treatment of post-traumatic stress disorders), the in-vitro and in-vivo behaviors of SLNURB597 were further investigated.

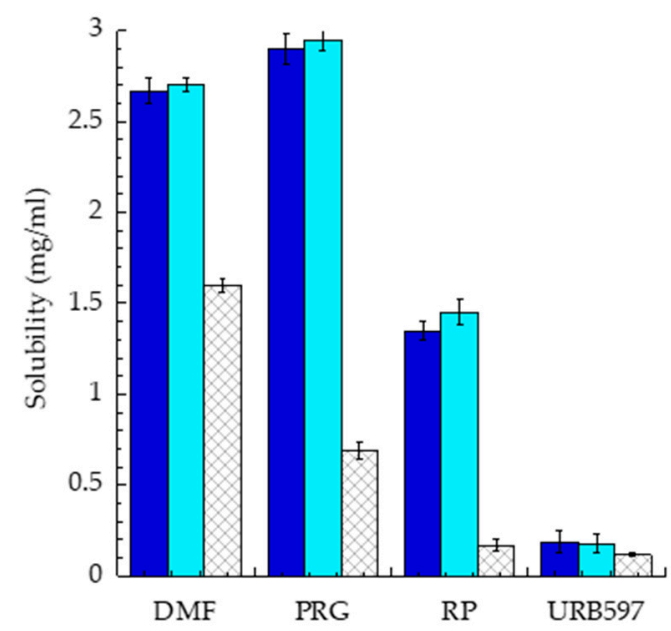

Figure 2. Comparative analysis of drug solubility in SLN (blue), NLC (light blue). and PEG 400/P80/saline 5:5:90 (v/v/v) (criss-cross). Adapted from [1].

\subsection{In Vitro Release Kinetics of URB597 from SLN}

The potential of SLN/P80 as delivery systems of URB597 was investigated with respect to a drug solution in PEG 400/P80/saline and a drug suspension in poloxamer 188 $(2.5 \% w / w)$, taken as controls. To this aim, the in vitro drug release profiles from SLN/P80URB597 was determined by a dialysis method. As reported in Figure 3, the URB597 release kinetic from SLN/P80 was almost superposable to that from PEG 400/P80/saline, while, as expected, the release of URB597 in the suspension form was reduced, especially in the slower phase, occurring $3 \mathrm{~h}$ after starting the experiment. 


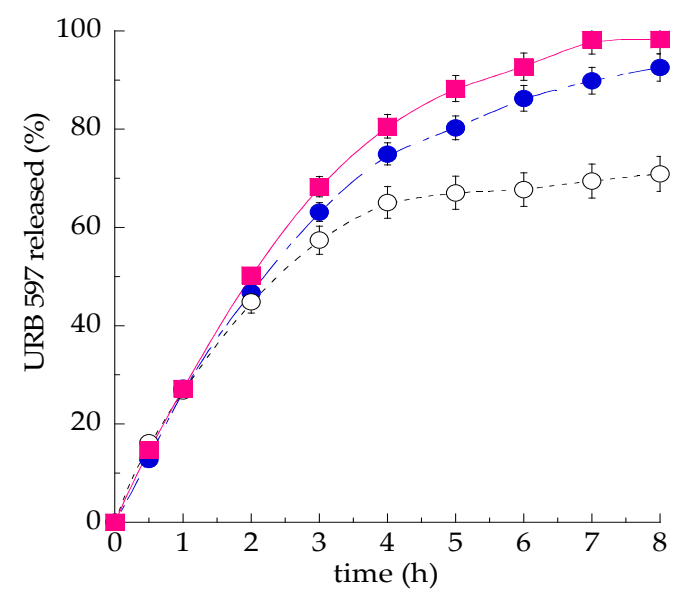

Figure 3. In vitro release kinetics of URB597 from SLN/P80-URB597 (blue circles), poloxamer 188 $(2.5 \% w / w)$ (white circle) and PEG 400/P80/water 5:5:90 (v/v/v) (pink square). Experiments were performed by dialysis method. Data are the mean of 6 experiments \pm S.D. Adapted from [1].

\subsection{In Vivo Behavioral Test}

The efficacy of SLN/P80-URB597 on brain function has been investigated in rats by behavioral tests. Some researchers have found that i.p. administration of the anandamide hydrolysis inhibitor URB597, dissolved in PEG400/P80/saline, induces prosocial effects in rats, increasing social play $[9,10]$ and correcting the social and communicative deficits in an animal model of autism spectrum disorders [14]. In this study, as an alternative to i.p. administration, the non-invasive i.n. administration route was proposed. Indeed, the latter allows to directly transport drugs to the brain through olfactory nerve, by-passing the BBB [15]. Particularly SLN/P80-URB597 administered by i.n. route induced behavioral effects comparable to those induced by i.p. injection of URB597 dissolved in PEG400/P80/saline (data not shown), confirming the suitability of the i.n. route to address the therapeutic potential of anandamide hydrolysis inhibitors in social dysfunctions, such as autism.

\section{Conclusions}

The present study suggests the suitability of lipid nanoparticles for solubilization and administration of poorly soluble drugs, avoiding unphysiological solvents or solvent mixtures. Notably, lipid nanoparticles enable four- and eight-fold increases in RP and PRG solubility with respect to PEG400/P80/saline, usually employed for in vivo preclinical studies. Moreover, SLN/P80-URB597 administered by i.n. route appears a non-invasive effective strategy to treat social dysfunctions.

Author Contributions: E.E., R.C. and C.N. conceived and designed the experiments; M.S. and V.T. performed the experiments; E.E., V.T. and R.C. analyzed the data; M.D. and P.M. conducted cryoTEM and X ray diffraction analyses; E.E. and C.N. wrote the paper. All authors have read and agreed to the published version of the manuscript.

Institutional Review Board Statement: The study was conducted according to the guidelines of the Declaration of Helsinki, approved by the Italian Ministry of Health (Rome, Italy) and performed in agreement with the guidelines released by the Italian Ministry of Health (D.L. 26/14) and the European Community Directive 2010/63/EU.

Acknowledgments: This study was funded his work was funded by “FIRB 2010. Fondo per gli Investimenti della Ricerca di Base" from the Ministry of the University and Research of Italy (code RBFR10XKHS) and by Unife FIR2018.

Conflicts of Interest: The authors declare no conflict of interest. 


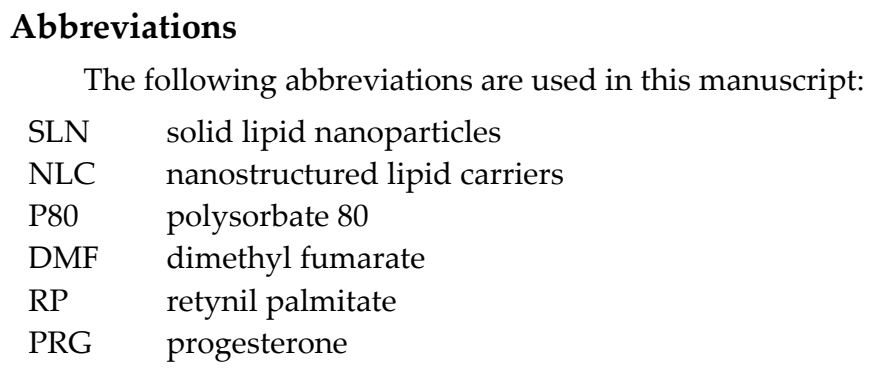

\section{References}

1. Esposito, E.; Drechsler, M.; Mariani, P.; Carducci, F.; Servadio, M.; Melancia, F.; Ratano, P.; Campolongo, P.; Trezza, V.; Cortesi, R.; et al. Lipid nanoparticles for administration of poorly water soluble neuroactive drugs. Biomed. Microdevices 2017, 19, 44-58, doi:10.1007/s10544-017-0188-x.

2. Pires, P.C.; Santos, L.T.; Rodrigues, M.; Alves, G.; Santos, A.O. Intranasal fosphenytoin: The promise of phosphate esters in nose-to-brain delivery of poorly soluble drugs. Int. J. Pharm. 2020, 120040, doi:10.1016/j.ijpharm.2020.120040.

3. Vo, C.L.-N.; Park, C.; Lee, B.-J. Current trends and future perspectives of solid dispersions containing poorly water-soluble drugs. Eur. J. Pharm. Biopharm. 2013, 85, 799-813, doi:10.1016/j.ejpb.2013.09.007.

4. Mu, H.; Holma, R.; Müllertz, A. Lipid-based formulations for oral administration of poorly water-soluble drugs. Int. J. Pharm. 2013, 453, 215-224, doi:10.1016/j.ijpharm.2013.03.054.

5. Esposito, E.; Sguizzato, M.; Drechsler, M.; Mariani, P.; Carducci, F.; Nastruzzi, C.; Valacchi, G.; Cortesi, R. Lipid nanostructures for antioxidant delivery: A comparative preformulation study. Beilstein J. Nanotechnol. 2019, 10, 1789-1801, doi:10.3762/bjnano.10.174.

6. Esposito, E.; Ravani, L.; Drechsler, M.; Mariani, P.; Contado, C.; Roukolainen, P.; Ratano, P.; Campolongo, P.; Trezza, V.; Nastruzzi, C.; et al. Cannabinoid antagonist in nanostructured lipid carriers (NLC): Design, characterization and in vivo study. Mater. Sci. Eng. C 2015, 48, 328-336, doi:10.1016/j.msec.2014.12.012.

7. Esposito, E.; Drechsler, M.; Cortesi, C.; Nastruzzi, C. Encapsulation of cannabinoid drugs in nanostructured lipid carriers. Eur. J. Pharm. Biopharm. 2016, 102, 87-91, doi:10.1016/j.ejpb.2016.03.005.

8. Pecora, R. Dynamic light scattering measurement of nanometer particles in liquids. J. Nanopart. Res. $2000,2,123-131$.

9. Manduca, A.; Servadio, M.; Campolongo, P.; Palmery, M.; Trabace, L.; Vanderschuren, L.J.; Cuomo, V.; Trezza, V. Strain- and context-dependent effects of the anandamide hydrolysis inhibitor URB597 on social behavior in rats. Eur. Neuropsychopharmacol. 2014, 24, 1337-1348, doi:10.1016/j.euroneuro.2014.05.009.

10. Trezza, V.; Vanderschuren, L.J.M.J. Bidirectional cannabinoid modulation of social behavior in adolescent rats. Psychopharmacology 2008, 197, 217-227, doi:10.1007/s00213-007-1025-3.

11. Liu, Y.; Qiu, J.; Wang, Z.; You, W.; Wu, L.; Ji, C.; Chen, G. Dimethylfumarate alleviates early brain injury and secondary cognitive deficits after experimental subarachnoid hemorrhage via activation of Keap1-Nrf2-ARE system. J. Neurosurg. 2015, 123, 915-923, doi:10.3171/2014.11.JNS132348.

12. Brotfain, E.; Gruenbaum, S.E.; Boyko, M.; Kutz, R.; Zlotnik, A.; Klein, M. Neuroprotection by estrogen and progesterone in traumatic brain injury and spinal cord injury. Curr. Neuropharmacol. 2016, 14, 641-653, doi:10.2174/1570159 × 1466616030912355 .

13. Bitarafan, S.; Mohammadpour, Z.; Jafarirad, S.; Harirchian, M.-H.; Yekaninejad, M.S.; Saboor-Yaraghi, A.A. The effect of retinylpalmitate on the level of pro and anti-inflammatory cytokines in multiple sclerosis patients: A randomized double blind clinical trial. Clin. Neurol. Neurosurg. 2019, 177, 101-105, doi:10.1016/j.clineuro.2019.01.003.

14. Servadio, M.; Melancia, F.; Manduca, A.; di Masi, A.; Schiavi, S.; Cartocci, V.; Pallottini, V.; Campolongo, P.; Ascenzi, P.; Trezza, $\mathrm{V}$. Targeting anandamide metabolism rescues core and associated autistic-like symptoms in rats prenatally exposed to valproic acid. Transl. Psychiatry 2016, 6, e902, doi:10.1038/tp.2016.182.

15. Kashyap, K.; Shukla, R.R. Drug Delivery and Targeting to the Brain Through Nasal Route: Mechanisms, Applications and Challenges. Curr. Drug Deliv. 2019, 16, 887-901, doi:10.2174/1567201816666191029122740. 